\title{
Scaffolds for Intracerebral Grafting of Neural Progenitor Cells After Cerebral Infarction:A Systematic Review
}

\author{
Laura K Cohen ${ }^{1}$ and Matthew B Jensen,", \\ ${ }^{1}$ Johns Hopkins University, School of Medicine, Baltimore, USA \\ 2 Department of Neurology, University of Wisconsin, Madison, USA \\ *Corresponding author: Matthew B Jensen, Department of Neurology, University of Wisconsin, Madison, USA. Tel:+608-2654351, Fax: +608-2630412, E-mail: jensen@neurology.wisc.edu
}

Received: November 22, 2014; Revised: December 29, 2014; Accepted: January 10, 2015

\begin{abstract}
Context: Intracerebral grafting of neural progenitor cells is a promising potential treatment to improve recovery after stroke, but the structural disruption and cavitation of brain tissue that occurs creates an unfavorable environment for graft cell survival. To overcome this obstacle, scaffold materials have been used as extracellular matrix to provide structural support for the transplanted cells. Many materials could potentially be used as scaffolds for this application.

Evidence Acquisition: We performed a systematic review to determine the available evidence supporting specific scaffolds for neural progenitor cell grafting after stroke. Articles were identified with a MeSH search on PubMed. Relevant references and "related articles" of selected manuscripts were also reviewed. Full original articles published prior to May 2013 presenting unique experimental data describing intracerebral grafting of neural progenitor cells in a scaffold after cerebral infarction were included in our study. All selected articles were reviewed thoroughly by the authors for relevant data.

Results: We found reports of use of scaffolds composed of polyglycolic acid, poly [lactic-co-glycolic acid] particles (with and without VEGF), hyaluronan-heparin-collagen hydrogel, Matrigel, collagen and extracellular matrix derived from porcine brain and urinary bladder. While multiple beneficial effects were reported, the optimal scaffold is unclear as we found no direct comparisons.

Conclusions: We conclude that multiple scaffolds appear promising for neural progenitor cell grafting after stroke, but further research is needed to optimize this neurorestorative approach. Thus, we hope to provide a basic understanding of the state of scaffolds for neural progenitor cell grafting after stroke and to encourage further research. Based on the methods of the discussed studies, we propose a standardized set of outcomes that would best be used to evaluate and compare the effectiveness of a given scaffold.
\end{abstract}

Keywords: Scaffold; Bio Scaffold; Neural Progenitor Cells; Grafting; Extracellular Matrix; Hydrogel; Neural Stem Cells; Stroke; Transplantation

\section{Context}

Post-stroke disability is an enormous public health problem. The majority of the 800,000 annual stroke victims in the United States survive the brain injury, but most stroke survivors are left with some degree of functional impairment, making it the leading cause of adult disability with over four million Americans currently suffering from poststroke disability (1-3). Replacement of lost brain neurons by intracerebral grafting of human neural progenitor cells is a promising potential therapy to improve stroke recovery (4). A major obstacle to translation of this treatment from the laboratory to clinical trials is the structural disruption and cavitation of brain tissue that occurs with stroke, which creates an unfavorable environment for graft cell survival. The infarcted tissue often disintegrates leaving a cavity that lacks the necessary structural support and perfusion for successful cell grafting.

To overcome this obstacle, researchers have investigated the use of structurally supportive materials as extracellu- lar matrix termed scaffolds, in which neural progenitors can be transplanted to promote cell survival and integration into the brain (5). In addition to the desired function of graft cell support, the molecular composition and other properties of certain scaffolds might also have beneficial effects on important host tissue responses, including inflammation, astrocytosis, and angiogenesis (6). There are many scaffolds available that may be useful for this application, but the safety, efficacy and ease of use of these materials likely varies. We, therefore, sought to review the available evidence of scaffolds that have been used for intracerebral grafting of neural progenitor cells after cerebral infarction. Further, in reviewing the available studies that vary widely in their focus and set of outcomes, we sought to compile and share the information that exists regarding scaffold use and to suggest an avenue of research that could be pursued with a standard set of outcomes to best determine the efficacy of given scaffolds for this purpose. 


\section{Evidence Acquisition}

\subsection{Data Sources}

We searched PubMed in May 2013 using the search terms: ("neurons" [MeSH Terms] or "neurons" [All Fields] or ("neural" [All Fields] and "cell" [All Fields]) or "neural cell" [All Fields]) and (vehicle [All Fields] or scaffold [All Fields] or gel [All Fields] or ("Matrix" [Journal] or "Matrix Suppl" [Journal] or "matrix" [All Fields])) and ("stroke" [MeSH Terms] or "stroke" [All Fields]).

\subsection{Study Selection}

We included articles in English published prior to May 2013 of unique experimental data that described intracerebral grafting of neural progenitor cells in a scaffold after cerebral infarction. We excluded abstracts. Titles, abstracts, or full articles were reviewed to determine if each search result matched our selection criteria. We also reviewed the references and "related articles" of the selected articles and review articles found with our search for additional matching articles.

\subsection{Data Extraction}

All selected articles were reviewed thoroughly by the authors for relevant data.

\section{Results}

Our search returned 610 results, of which three met our selection criteria. We found five further matching articles in the references and "related articles" of the selected articles and review articles found with our search.

In 2002, Park et al. (7) reported the use of a woven net of polyglycolic acid fibers as a scaffold for intracerebral grafting of mouse neural progenitor cells in a mouse model of focal hypoxic-ischemic brain injury. The solid cell-scaffold construct was created in vitro, the exact method of implantation into the infarct seven days after infarction was unclear, but involved a craniotomy of unstated size and a glass micropipette. Mice with the cell-scaffold construct implanted in the infarct were compared with those implanted with scaffold alone, cells alone, or a liquid vehicle alone. The authors qualitatively described that the cell scaffold implant filled the lesion cavity, and that host cells and blood vessels infiltrated the degrading scaffold. Surviving graft cells were found up to three months after transplantation, some of which labeled for markers of neurons, astrocytes and oligodendrocytes; graft cell survival and differentiation was not quantified. Infarct cavity size, astrocytosis and inflammation were qualitatively stated to be diminished in the cell-scaffold group. Axonal tracing suggested that graftderived axons projected through the corpus callosum to the contralateral hemisphere in the cell-scaffold group. The authors qualitatively mentioned improved behav- ioral recovery in the cell-scaffold group.

In 2009, Bible et al. (8) reported the use of poly [lacticco-glycolic acid] (PLGA) particles coated with allylamine and fibronectin as a scaffold for intracerebral grafting of mouse neural progenitor cells in a rat model of ischemic stroke. The cell-seeded scaffold particles were created in vitro and injected into the infarct two weeks after infarction; there was no control group. Scaffold degradation and surviving graft cells labeling for markers of neural progenitors, neurons, or astrocytes was qualitatively described after the seven-day survival period; graft cell survival and differentiation was not quantified. Minimal astrocytosis, inflammation and angiogenesis were qualitatively described. Behavior testing was not reported, but magnetic resonance imaging data of cell and scaffold imaging characteristic was presented.

In 2010, Yu et al. (9) reported the use of collagen type I as a scaffold for neural progenitor cells following a rat model of ischemic injury. Twenty four hour after the two hour middle cerebral artery occlusion injury rats received injection of neural stem cells (NSCs) alone, collagen alone, NSCs + collagen, or vehicle, or alternatively were subjected to sham surgery. The researchers found that neurologic function, as measured by the neurological severity score, was significantly improved in both NSC treatment groups (NSCs alone and NSCs + collagen) as compared to the media injected groups' scores. There was no significant difference in functional recovery between the two NSC treatment groups. However, the graft cells in the NSCs + collagen transplant group differentiated into neurons significantly more than those in the NSCs transplant group. The authors stated that the collagen began to degrade within 7 days of transplant and had fully degraded by 30 days post-transplant. Finally, the authors mentioned that synapse formation was present and significantly more abundant than in the control group, at 30 days post-transplant in the NSCs + collagen transplant animals although they did not compare this to other treatment groups. Inflammation, astrocytosis, angiogenesis, infarct size, and quantification of cell survival were not discussed.

In 2010, Jin et al. (10) reported the use of Matrigel as a scaffold for intracerebral grafting of human neural progenitor cells in a rat model of ischemic stroke. The rats were administered the following into the infarct three weeks after infarction: liquid vehicle alone, liquid Matrigel alone (which then gelled in the infarct), cells alone, cells in liquid Matrigel, or cells cultured and transplanted in solid Matrigel. All were administered by injection except the solid cell-scaffold group which has a craniotomy of unstated size and insertion of the cell-scaffold construct with a glass micropipette. Behavioral testing during the four to nine week survival period showed that the solid cell-scaffold group recovered better than the vehicle group, but worse than the sham (no infarct) animals. Both the liquid and solid cell-scaffold groups had smaller infarcts. The solid cell-scaffold group had better graft cell 
Cohen LK et al.

survival than the liquid cell-scaffold group, which in turn had better graft cell survival than the cells alone group. Graft cell neuronal differentiation was increased in the solid cell-scaffold group, with some producing action potentials. Small numbers of graft cells expressed markers of neural progenitor cells or astrocytes, which was not different between groups. Scaffold degradation, astrocytosis, inflammation and angiogenesis were not described.

In 2010, Zhong et al. (11) reported the use of a hydrogel of hyaluronan, heparin and collagen as a scaffold for intracerebral grafting of mouse neural progenitors (NP) cells, derived from embryonic stem (ES-NP) cells or embryonic cortical (EC-NP) cells, in a mouse model of ischemic stroke. The mice had cells injected into the infarct seven days after infarction in four groups: ES-NP cells alone, ECNP cells alone, ES-NP cells in scaffold and EC-NP cells in scaffold were compared with those implanted with scaffold alone. After a two-week survival period both cell lines had a two-fold increase in graft cell survival with the scaffold, however EC-NP cells survived better than ES-NP cells with or without a scaffold. EC-NP cells mostly expressed astrocytic markers, while no expression of mature neural cell markers was seen with the ES-NP cells. The scaffold had no effect on graft cell differentiation with either cell line. The scaffold decreased the infiltration of activated microglia and macrophages into the graft. No effect of the scaffold was seen on angiogenesis or reactive astrocytosis. Scaffold degradation and behavioral testing were not reported.

In 2010, Jin et al. (12) reported the use of Matrigel as a scaffold for NPCs in a study meant to confirm their earlier 2010 results in a group of aged rats following ischemic injury to better model aging stroke patients. NPCs and Matrigel or a vehicle were transplanted into rats (no matrigel-only or NPC-only controls were used as in their earlier study) three weeks after MCAO. Rats were either 3 month or 24 month old at stroke onset. Results confirmed the positive outcomes seen in the previous study in both 3 month and 24 month rats. Graft cells were seen to survive and continue to divide, although some cells underwent cell death. Immunohistochemistry qualitatively showed differentiation into both astroglial cells and neurons. Results held up in this study and behavioral function and infarct volume in the 24 month group, while worse than the 3 month group immediately following injury, were significantly improved by the NPC + Matrigel transplant. Quantitative cell survival and differentiation and data regarding inflammation, astrocytosis, angiogenesis, and scaffold degradation were not reported.

In 2012, Bible et al.(13) reported the use of a gelling form of extracellular matrix derived from porcine brain and urinary bladder as a scaffold for intracerebral grafting of human neural progenitor cells in a rat model of ischemic stroke. Rats with cell-scaffold suspension injected into the infarct two weeks after infarction were compared with those implanted with scaffold alone. Surviving graft cells as well as minimal astrocytosis and inflammation were qualitatively described after the seven-day survival period. Scaffold degradation, graft cell differentiation, angiogenesis and behavioral testing were not reported, but magnetic resonance imaging did not suggest restoration of normal striatal tissue imaging characteristics.

In 2012, Bible et al. (14) reported the use of PLGA particles as a scaffold for NSCs, with or without added VEGF. The researchers sought to promote true de novo tissue formation by addition of VEGF because vasculature had not been observed in their 2009 study. They determined that the addition of VEGF increased the number of endothelial cells (ECs) that were recruited to the injured area. PLGA + NSC transplant increased ECs, but the addition of VEGF promoted higher numbers of ECs and the beginning of neo-vascularization, qualitatively observed by formation of tube-like structures likely representing new blood vessels. Data regarding functional outcomes, scaffold degradation, cell survival and differentiation, infarct volume, inflammation and astrocytosis were not reported.

We found no studies directly comparing different scaffolds for intracerebral grafting of neural progenitor cells after cerebral infarction.

\section{Conclusions}

The optimal scaffold for use in cell therapy after stroke is unclear, as we found no direct comparisons. We conclude that multiple scaffolds appear promising for intracerebral grafting of neural progenitor cells after cerebral infarction, but further research, in particular direct comparison studies of different scaffolds, is needed to optimize this neurorestorative approach.

Thus far, the use of scaffolds for intracerebral grafting of neural progenitor cells after ischemic injury has not been a major focus of the field. As this review shows the approaches vary widely and the crosstalk and consensus is not there to determine which approach to scaffolding might be most effective. Therefore, we would suggest a standard set of outcomes for future studies of scaffolds for cell grafting after stroke. The following should be included, whether in investigating a single scaffold or in comparing a variety of types: quantification of graft cell survival and differentiation, infarct size, host response (inflammation, astrocytosis, angiogenesis), functional/ behavioral outcomes and description of scaffold degradation or lack thereof. We hope with this review to collect and present the existing information and to encourage further research in this field.

We found evidence suggesting that multiple scaffold materials may improve graft cell and host tissue outcomes with intracerebral transplantation of neural progenitor cells in animal stroke models, but no reports of direct comparisons.

In 2002 Park et al. (7) qualitatively described multiple improved graft cell and host tissue outcomes with the use of a solid polyglycolic acid scaffold when compared to control groups. While the limited method details and 
results quantification complicated replication and comparison to other studies, the demonstrated feasibility and qualitative statement of improved behavioral recovery with this approach is encouraging.

In 2009 Bible et al. (8) also qualitatively described multiple positive graft cell and host tissue outcomes with the use of PLGA particles as a scaffold. This approach has the advantage of the ability to inject the cell-seeded scaffold particles through a needle, which could potentially improve safety over solid implants by minimizing the required craniotomy size and injury of healthy brain tissue during grafting. The lack of a control group precludes the ability to draw conclusions about the effect of the scaffold on graft cell and host tissue outcomes, but the demonstrated feasibility and description of imaging characteristics are useful.

In 2010, Yu et al. (9) presented qualitative and controlled data showing improvements in functional recovery and cell behavior with the use of Collagen Type I. The cellular differentiation data suggested that Collagen (when combined with NSCs) had a positive impact as compared to NSCs alone (or Collagen alone) and increased neuronal differentiation of graft cells. However, while the functional recovery seen in both NSC groups is promising, the lack of a significant difference in functional recovery between the NSCs alone group and the NSCs + Collagen group means that functional outcomes need to be further explored to determine if Collagen could be a necessary or effective scaffold.

In 2010 Jin et al. (10) presented quantitative and controlled data showing multiple improved graft cell and host tissue outcomes with the use of another commercially available hydrogel scaffold. Matrigel is commonlyused to study three-dimensional cell culture, and also allows for injection through a needle followed by gelling in situ. While they found beneficial effects of the scaffold with this approach, even greater benefit was found for some outcomes when the cells were implanted with Matrigel that had already gelled in vitro. These outcomes included decreased infarct size, increased graft cell survival, and increased graft cell neuronal differentiation, including a proportion of graft cells capable of firing action potentials. Most importantly, these graft cell and host tissue outcomes were translated into improved behavioral recovery of the groups administered cells in the scaffold. Replication of this approach could also provide additional useful information such as the rate and extent of scaffold degradation, as well as effects on host tissue astrocytosis, inflammation, and angiogenesis, which could allow insights into the mechanism of the beneficial effects seen with the use of the scaffold.

In 2010 Zhong et al. (11) presented quantitative and controlled data showing multiple improved graft cell and host tissue outcomes with the use of a commercially available hydrogel scaffold predominantly composed of hyaluronan. This approach combines the advantages of needle injections of cells and the scaffold, followed by in situ gelling, with simpler material preparation procedures. The beneficial effects of the scaffold were similar with neural progenitor cells derived from both embryonic stem cells and embryonic cortical cells, adding confidence to the generalizability of the findings. Replication of this approach with the addition of behavioral testing could allow us to also see if the beneficial effects of the scaffold on graft cell and host tissue outcomes also lead to improved functional recovery.

In 2010, Jin et al. (12) presented quantitative and controlled data that further confirmed their earlier conclusions with the use of Matrigel. The encouraging results suggested not only that their data was reproducible, but also that the effect seen of the NPC + Matrigel transplant was generalizable, specifically to the aged population, generally the target of these stroke therapies. Quantitative analysis of the graft cell behavior and host tissue response would further add to solidify the argument for Matrigel as a scaffold.

In 2012 Bible et al. (13) presented controlled data with qualitative descriptions of multiple positive graft cell and host tissue outcomes with the use of a hydrogel scaffold derived from animal tissues. This demonstration of feasibility adds an additional promising scaffold material to the growing armamentarium for this approach, and the imaging methods described provide further tools for in vivo monitoring of graft cell and host tissue effects of the scaffolds.

In 2012, Bible et al. (14) presented quantitative and controlled data that showed an improvement in their scaffold design from 2009 with the use of PLGA particles supplemented with VEGF. The lack of neo-vascularization in the 2009 study and the subsequent addition of the vascularization-promoting protein VEGF suggested that a structural component might not be the only factor present in the most promising scaffold. The study allows for the consideration of supplementing structural scaffolding components with proteins or other molecules to best achieve recovery.

To our knowledge, this is the first systematic review of scaffolds for intracerebral grafting of neural progenitor cells after cerebral infarction, but it has several weaknesses. We found few published articles of studies on this topic. This may be accurate because this specific area of research is new, or it may be due to a poor ability to capture all applicable publications due to limitations of article indexing or referencing by the selected articles. In either case, unfortunately, we are left with a fairly limited view of the possibilities that this field has to offer, and nothing in the way of direct comparisons. Rather than discouragement, however, this should encourage researchers that this early work shows promising results while there is yet much to be learned.

The optimal scaffold for use in cell therapy after stroke is unclear, as we found no direct comparisons. We conclude that multiple scaffolds appear promising for intracerebral grafting of neural progenitor cells after cerebral 
infarction, but further research, in particular direct comparison studies of different scaffolds, is needed to optimize this neurorestorative approach.

Thus far, the use of scaffolds for intracerebral grafting of neural progenitor cells after ischemic injury has not been a major focus of the field. As this review shows the approaches vary widely and the crosstalk and consensus is not there to determine which approach to scaffolding might be most effective. Therefore, we would suggest a standard set of outcomes for future studies of scaffolds for cell grafting after stroke. The following should be included, whether in investigating a single scaffold or in comparing a variety of types: quantification of graft cell survival and differentiation, infarct size, host response (inflammation, astrocytosis, angiogenesis), functional/ behavioral outcomes, and description of scaffold degradation or lack thereof. We hope with this review to collect and present the existing information and to encourage further research in this field.

\section{Acknowledgements}

We appreciate the editorial advice of Crystal Hall, Cassandra Heilingoetter, Lindsey Jager, Rajeev KrishnaneyDavison, Susanna Kwok, Jin Kwon, Wai Yin Leung, Peter Ostergaard, Malissa Roberts, Natanya Russek and Kelly Wallin.

\section{Authors' Contributions}

Study concept and design: Laura K. Cohen, Matthew B. Jensen. Acquisition of data: Laura K. Cohen. Analysis and interpretation of data: Laura K. Cohen, Matthew B. Jensen. Drafting of the manuscript: Laura K. Cohen. Critical revision of the manuscript for important intellectual content: Laura K. Cohen, Matthew B. Jensen. Study supervision: Matthew B. Jensen.

\section{Funding/Support}

This study was supported in part by grant K08NS079622$01 \mathrm{~A} 1$ from the national institutes of health and by student research fellowship 12UFEL12030172 from the American heart association.

\section{References}

1. Benowitz LI, Carmichael ST. Promoting axonal rewiring to improve outcome after stroke. Neurobiol Dis. 2010;37(2):259-66.

2. Gilman S. Time course and outcome of recovery from stroke: relevance to stem cell treatment. Exp Neurol. 2006;199(1):37-41.

3. Roger VL, Go AS, Lloyd-Jones DM, Adams RJ, Berry JD, Brown TM, et al. Heart disease and stroke statistics-2011 update: a report from the American Heart Association. Circulation. 2011;123(4):e18-e209.

4. Bliss TM, Andres RH, Steinberg GK. Optimizing the success of cell transplantation therapy for stroke. Neurobiol Dis. 2010;37(2):275-83.

5. Delcroix GJ, Schiller PC, Benoit JP, Montero-Menei CN. Adult cell therapy for brain neuronal damages and the role of tissue engineering. Biomaterials. 2010;31(8):2105-20.

6. Potter W, Kalil RE, Kao WJ. Biomimetic material systems for neural progenitor cell-based therapy. Front Biosci. 2008;13:806-21.

7. Park KI, Teng YD, Snyder EY. The injured brain interacts reciprocally with neural stem cells supported by scaffolds to reconstitute lost tissue. Nat Biotechnol. 2002;20(11):1111-7.

8. Bible E, Chau DY, Alexander MR, Price J, Shakesheff KM, Modo M. The support of neural stem cells transplanted into stroke-induced brain cavities by PLGA particles. Biomaterials. 2009;30(16):2985-94.

9. Yu H, Cao B, Feng M, Zhou Q, Sun X, Wu S, et al. Combinated transplantation of neural stem cells and collagen type I promote functional recovery after cerebral ischemia in rats. Anat Rec (Hoboken). 2010;293(5):911-7.

10. Jin K, Mao X, Xie L, Galvan V, Lai B, Wang Y, et al. Transplantation of human neural precursor cells in Matrigel scaffolding improves outcome from focal cerebral ischemia after delayed postischemic treatment in rats. J Cereb Blood Flow Metab. 2010;30(3):534-44.

11. Zhong J, Chan A, Morad L, Kornblum HI, Fan G, Carmichael ST. Hydrogel matrix to support stem cell survival after brain transplantation in stroke. Neurorehabil Neural Repair. 2010;24(7):636-44.

12. Jin K, Mao X, Xie L, Greenberg RB, Peng B, Moore A, et al. Delayed transplantation of human neural precursor cells improves outcome from focal cerebral ischemia in aged rats. Aging Cell. 2010;9(6):1076-83.

13. Bible E, Dell'Acqua F, Solanky B, Balducci A, Crapo PM, BadylakSF, et al. Non-invasive imaging of transplanted human neural stem cells and ECM scaffold remodeling in the stroke-damaged rat brain by (19)F- and diffusion-MRI. Biomaterials. 2012;33(10):2858-71.

14. Bible E, Qutachi O, Chau DY, Alexander MR, Shakesheff KM, Modo M. Neo-vascularization of the stroke cavity by implantation of human neural stem cells on VEGF-releasing PLGA microparticles. Biomaterials. 2012;33(30):7435-46. 